Modern Physics Letters B, Vol. 17, Nos. 10, 11 \& 12 (2003) 649-656

(C) World Scientific Publishing Company

\title{
POINT NORMAL METAL-SUPERCONDUCTOR (NS) CONTACT IN NONBALLISTIC REGIME
}

\author{
I. N. ASKERZADE \\ Department of Physics, Ankara University, Tandogan, 06100 Ankara, Turkey \\ and Institute of Physics, Azerbaijan Academy of Sciences, \\ Baku-370143, Azerbaijan \\ solstphs@physics.ab.az \\ I. O. KULIK* \\ Department of Physics, Bilkent University, Bilkent, 06533 Ankara, Turkey
}

\begin{abstract}
We analyze the point NS contact conductivity taking into account the depression of superconductivity at high-injection current density and Andreev reflection at the adaptive NS boundary. The dependence of the excess current on the voltage, as well as conductivity of contact at arbitrary voltage is obtained.
\end{abstract}

\section{Introduction}

Andreev-reflection point contact spectroscopy is based on the measurements of the transformation of the quasiparticles to Cooper pairs, which happens at least at the distance of coherence length $\xi$ from the interface between a normal metal $N$ and superconductor $S .{ }^{1}$ Electronic transport in NS boundary is described by the Artemenko, Volkov and Zaitsev, ${ }^{2}$ Zaitsev $^{3}$ and by the BTK (Blonder-Tinkham-Klapwijk) formalism, ${ }^{4}$ which was developed for the ballistic junctions. In this regime a point contact consists of the normal metal $\mathrm{N}$ and superconductor $\mathrm{S}$, whose radius $r_{0}$ is significantly less than the mean free path $l$. The ratio of conductance inside and outside the gap voltage predicted by the BTK is a factor of 2 . However, fit of the experimental values gave much smaller ratios. ${ }^{5}$ Similar amplitude reduction have been observed in phonon point-contact experiments of short mean-free-part materials. ${ }^{6}$ The typical contact size estimation from recent point contact spectroscopy in different superconductors gives ${ }^{7,8} r_{0}=5-60 \mathrm{~nm}$. Effects of impurity scattering taken into account by including scattering region with size smaller than the electronic mean-free-parth. Theory of diffusive NS contact was developed in Ref. 2 and it was fist time shown that the zero-bias conductance of such contact $G_{N S}=G_{N N}$ in contrast to the ballistic case, when $G_{N S}=2 G_{N N}$. With development of new technique of producing well conrtrolled constrictions of nanoscale $\operatorname{size}^{9}$ it is important

${ }^{*}$ B. Verkin Institute for Low Temperature Physics and Engineering, National Academy of Sciences of Ukraine, Kharkov, 310164, Ukraine 
to develop the theory for the explanation quantitatively point contact spectrum of the materials with a short mean-free-part.

As soon as the radius of the contact becomes comparable with superconducting coherence length $\xi$, the nonequilibrium phenomena may occur in NS junctions. This can be lead to suppression of the gap by (a) nonequilibrium population of normal electrons and phonons with high energy, (b) penetration of the magnetic flux vortices generated by the current through the contact, (c) heating of the contact region. A number of interesting features in the current-voltage characteristics of $\mathrm{Ag} / \mathrm{Ta}$ junctions was reported in Ref. 10. For the explanation of this experimental data was suggested theory of nonequilibrium $N c N^{\prime} S$ point contact. ${ }^{11,12}$ In $N S$ point contact with "high energy" quasiparticle injection allowing for phonon production, a non-equilibrium situationis produced. Radius of nonequilibrium region increases with increasing voltage biase. However, in this calculations explicit expression for the excess current was not derived. Other modified variant of the $N c N^{\prime} S$ junctions was applied for the description of cuprate $N S$ junctions, where effect of a near pair potential suppression taken into account. ${ }^{13}$ Heating effects in $N S$ junctions at high voltages, which can lead to reduction or even destruction of superconducting order parameter discussied by Khlus and Omelyanchuk. ${ }^{14}$

It is quite natural to expect that in the case of nanoscale size point contacts even the moderate bias in the NS junctions well lead to destruction of the order parameter within the superconducting side by the high injection current, which exceeds depairing current. We assume that a normal half-sphere will be developed within the superconducting half-space by the increasing of the applied current (Fig. 1). Such model of a contact has previously been proposed, ${ }^{15,16}$ termed "spherical spread out" model which assumes that two metals are glued along the sphere of raduis $r_{0}$ resulting in one dimensional $\mathrm{r}$-dependent current density in both ( $\mathrm{N}$ and $\mathrm{S}$ ) sides of contact. It is a limiting form of the hyperbolic contact model ${ }^{17}$ with the thickness of connection $\Delta z \rightarrow 0$. In this paper we will investigate the influence of the above mentioned behavior on the differential conductance $G_{N S}(V)$ and excess current $I_{\text {exc }}$.

\section{Basic Equations}

As a model for the NS point contact we consider metal connection in the form of an orifice in an impenetrable screen, as descibed above, i.e. "orifice" is actually a spherical surface of surface $4 \pi r_{0}^{2}$ (Fig. 1). We will assume that the right side of contact is s-wave superconductor. At low voltages, when the condition $V<V_{c}=\Delta / e$ is satisfied, the whole voltage drops in the left side is

$$
V_{1}=R_{1} I
$$

where $R_{1}$ is the resistance of the left $\mathrm{N}$-side, which can be calculated (see below). When current density at the orifice will reach the depairing current density $j_{0}{ }^{18}$ 


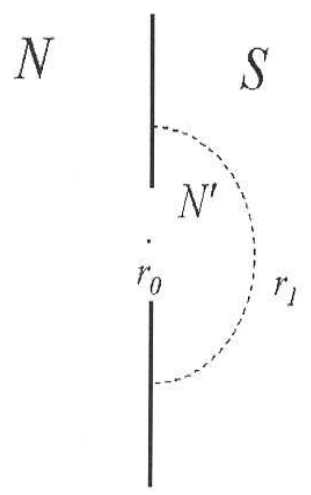

(a)

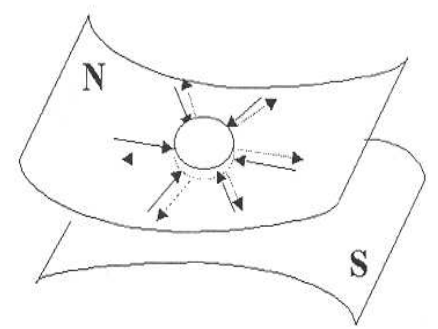

(b)

Fig. 1. (a) Sketch of the orifice-shaped point contact in impenetrable screen.Dotted line is an adaptive $N^{\prime} S$ boundary; (b) Schematic view of "spherical spread out" model of 2 d contact.

with total current

$$
I_{0}=4 \pi r_{0}^{2} j_{0}
$$

within the right superconducting half-space will develop a normal half-sphere, the center of which coincides with the center of the orifice. The radius of this half-sphere for the values of current $I>I_{0}$ is determined from

$$
I=4 \pi r^{2} j_{0}, \quad r=\left(\frac{I}{4 \pi j_{0}}\right)^{1 / 2} .
$$

Voltage drop in the right side is

$$
V_{2}=R_{2} I
$$

where $R_{2}$ is the resistance of the spherical sector. On the other hand, according to the Ohmic law (here considered that $l<r_{0}$, otherwise we have ballistic regime, for which Ohmic law is not applicable)

$$
V_{2}=\int E d r=\frac{I \rho_{2}}{2 \pi} \int \frac{d r}{r^{2}}=\frac{I \rho_{2}}{2 \pi}\left(r_{0}^{-1}-r_{1}^{-1}\right),
$$

where $\rho_{2}$ is the resistivity of the right half space in the normal state. For the left $\mathrm{N}$-space, using the last expression in the case when $r_{1} \rightarrow \infty$, we receive

$$
R_{1}=\frac{\rho_{1}}{2 \pi r_{0}},
$$

where $\rho_{1}$ is the resistivity of the left side. For the total voltage we receive the following expression:

$$
V=R_{M} I-\frac{\rho_{2}\left(4 \pi j_{0} I\right)^{1 / 2}}{2 \pi},
$$


where $R_{M}=\left(\rho_{1}+\rho_{2}\right)\left(1+Z^{2}\right) / 2 \pi r_{0}$ is so-called Maxwell resistance. The dimensionless parameter $Z$ is the normalized strength of the $\delta$ functional barrier localized in the orifice (a notation comprizing that of the BTK paper ${ }^{4}$ ). Last equation can be rewritten as

$$
i=\frac{\rho_{1} v}{\rho_{1}+\rho_{2}}+\left(\frac{\rho_{2}}{\rho_{1}+\rho_{2}}\right)^{2}\left(1+\left(1+2\left(\left(\frac{\rho_{1}}{\rho_{2}}\right)^{2}+\frac{\rho_{1}}{\rho_{2}}\right) v\right)^{1 / 2}\right),
$$

where the following notations are introduced : $i=I / I_{0}, v=V / R_{1} I_{0}\left(1+Z^{2}\right)$. As follows from Eq. (1), voltage $V_{c}=R_{1} I_{0}\left(1+Z^{2}\right)$. As can be seen from Eq.(8), even in the case of absence of Andreev reflection there is an excess current due to developing normal half-sphere within the SC half-space. For the differential conductance we receive:

$$
\frac{G_{N S}}{G_{N N}}=\frac{d i}{d v}=\frac{\rho_{1}}{\rho_{1}+\rho_{2}}\left(1+\frac{1}{\left(1+2\left(\frac{\rho_{1}^{2}}{\rho_{2}^{2}}+\frac{\rho_{1}}{\rho_{2}}\right) v\right)^{1 / 2}}\right) .
$$

In taking into account the Andreev reflection ${ }^{4}$ at the $N^{\prime} S$ boundary, the last formula can be rewritten as (we assume that the barrier strength at the $N^{\prime} S$ boundary is zero)

$$
\frac{G_{N S}}{G_{N N}}= \begin{cases}2 & \text { at } v<1 \\ \frac{\rho_{1}}{\rho_{1}+\rho_{2}}\left(1+\frac{1-\left(1-v^{-2}\right)^{1 / 2}}{1+\left(1-v^{-2}\right)^{1 / 2}}+\frac{1}{\left(1+2\left(\frac{\rho_{1}^{2}}{\rho_{2}^{2}}+\frac{\rho_{1}}{\rho_{2}}\right) v\right)^{1 / 2}}\right) & \text { at } v>1,\end{cases}
$$

where the second term in the curly brackets represents the Andreev contribution. ${ }^{4}$ As can be seen from the last equation at high voltage, $v \gg 1$, the behavior of the conductance is determined mostly by the non-Andreev contriburion since Andreev term rapidly decreases, as $v^{-2}$, in comparision with the third term in curly brackets. As follows from the last formula, conductance at $v \gg 1$ is determined by the ratio of resistances $\rho_{1} / \rho_{2}$. As pointed out in Ref. 4 , excess current in the case of zerobarrier strength due to Andreev reflection in $N^{\prime} S$ boundary can be calculated from the following formula

$$
I_{e x c}=\frac{1}{e R_{M}} \int_{0}^{\infty} A(E) d E=\frac{\pi \Delta}{2 e R_{M}},
$$

where $A(E)$ is the probability of Andreev reflection. ${ }^{4}$ For the total excess current we can get

$$
I_{e x c}=\frac{\pi \Delta}{2 e R_{M}}+I_{0}\left(1+\left(1+2\left(\left(\frac{\rho_{1}}{\rho_{2}}\right)^{2}+\frac{\rho_{1}}{\rho_{2}}\right) v\right)^{1 / 2}\right)\left(\frac{\rho_{2}}{\rho_{1}+\rho_{2}}\right)^{2} .
$$

As shown by the expression (8), non-Andreev contribution to the excess current depends on the applied voltage to the NS junctions. 


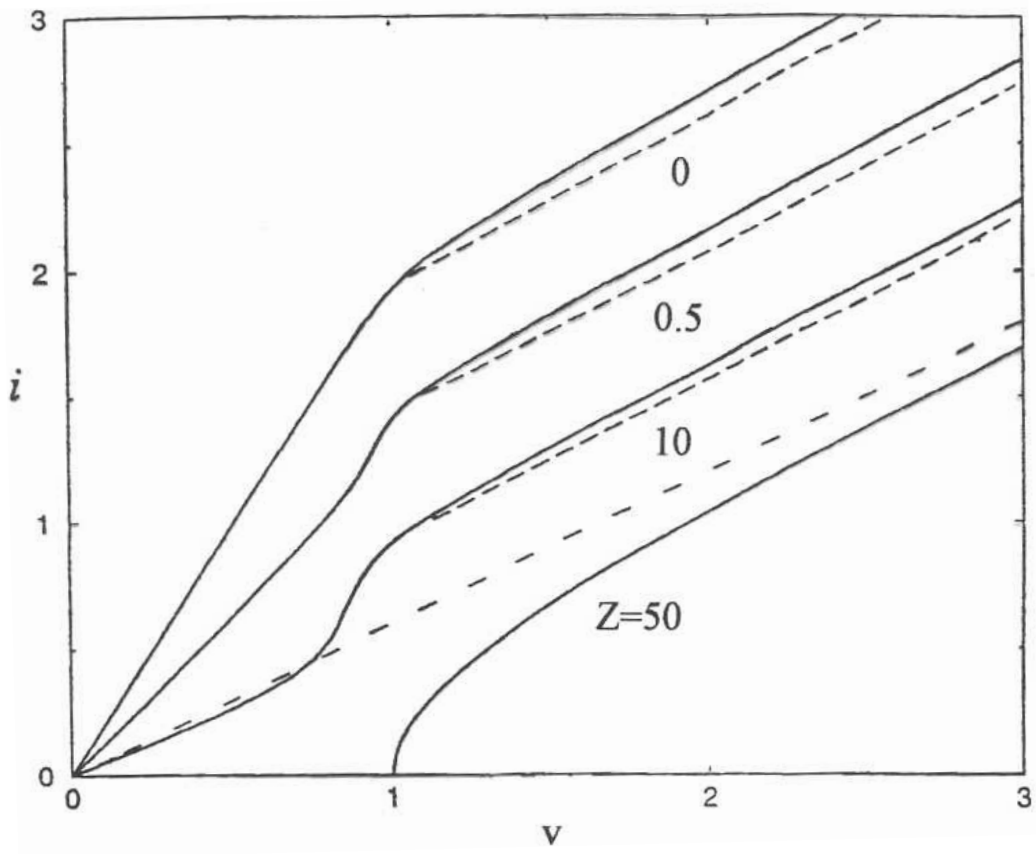

Fig. 2. Current versus voltage for various barrier strengths $Z=50,10,0.5,0$ (from bottom to top, dashed line marks the $N N^{\prime}$ case) at $\kappa \approx 1$ and $T=0$.

\section{Discussion}

We have proposed a simple theory for the point NS nonballistic junction. It is shown that total excess current is represented as the sum of two contributions: Andreev reflection at the adaptive (i.e. moving toward interior of superconductor at increasing current) $N^{\prime} S$ boundary and non-Andreev contribution, related to the developing N-half sphere in superconducting half-space. The calculated $I-V$ curve for the different barrier strength is presented in Fig. 2 for the parameter $\kappa \approx 1$ (see below). In general case, excess current depends on the applied voltage. At inceasing barrier strength, the excess current decreases. The latter result is directly accessible to experiment, and should be helpful in interpreting the wide variety of experimentally observed I-V curves.

In principle, the problem of conductance of a contact in the form of an orifice between two normal metals which differ one from another by the effective mass of carriers, free mean path and other physical properties, is not considered yet. In general case the calculation of the conductance of two dissimilar metals can be achieved with the help of the Landauer formula, or by using directly the Boltzmann approach. ${ }^{16}$ Early attempt for the calculation excess resistance in periodic NS structures with different resistivity of $\mathrm{N}$ and $\mathrm{S}$ layers was made by Atremenko, Volkov and Sergeev. ${ }^{19}$ Such theory in agreement with experimental data for the 
excess current of periodical systems of NS boundaries. ${ }^{20}$ However, influence of the dissimilarity of the two metals on the I-V curve, differential conductivity is not considered yet. From this point investigation nanoscale size nonballistic NS junctions with dissimilar electrodes is interesting. 
Here we develop simple phenomenological theory in which the dissimilarity of two metals is introduced as the ratio of resistivity of the left and right sides, $\kappa=$ $\rho_{1} / \rho_{2}$. This parameter strongly affects the non-Andreev excess current. For the case $\kappa \rightarrow \infty$, only Andreev excess current remains. In the opposite limit, $\kappa \rightarrow 0$, the non-Andreev excess current (in units of $I_{0}$ ) is constant, i.e. is equal approximately to 1 . For the case $\kappa \approx 1$, excess current increases two times only at $v \approx 14$. As can be seen from the expression for the differential conductance (10), in case $\kappa \rightarrow \infty$ the ratio of conductances inside and outside the gap voltage is a factor of 2 , as predicted by BTK theory. In the case of $\kappa \approx 1$, this ratio becomes smaller than 2 . Further decreasing of the parameter of dissimilarity, $\kappa$, leads to enhancement of the conductance ratio of NS nonballistic junction inside and outside the gap voltage. Comparision of the theorethical results with experimental data for nanoscale size NS junctions with dissimilar electrodes due to a lot of experiments seems very hard and may be presented in furute.

Thus, the analysis presented in this paper shows that $I-V$ curve of highcurrent density junction differs from that in ballistic NS junction. The formula for the excess current and differential conductance taking into account the developing of the normal half-sphere within the SC is obtained.

\section{References}

1. I. K. Yanson, in Symmetry and Pairing in Superconductors, Eds. M. Ausloos and S. Kruchinin (Kluwer Acad. Publ., 1999), pp. 271-285.

2. S. N. Artemenko, A. F. Volkov and A. V. Zaitsev, Solid State Communications 30, 771 (1979).

3. A. V. Zaitsev, Sov. Phys. JETP 51, 111 (1980); 52, 1018 (1980).

4. G. E. Blonder, M. Tinkham and T. M. Klapwjik, Phys. Rev. B25, 4515 (1982).

5. Y. E. Wilde, M. Iavarone, W. Welp, V. Metlushko, A. E. Koshelev, L. Aranson and G. W. Grabtee, Phys. Rev. Lett. 78, 4273 (1997).

6. I. K. Yanson, Sov. J. Low Temp. Phys. 9, 343 (1983).

7. L. F. Rybaltchenko, A. G. M. Jansen, P. Wyder, L. V. Tjutrina, P. C. Canfield, C. V. Tomy, D. McK. Paul, Physica C319, 189 (1999).

8. F. Laube, G. Goll, H. v. Lohneyesen and F. Lichtenberg, J. Low Temp. Phys. 117, 1575 (1999).

9. I. K. Yanson, in Quantum Mesoscopic Phenomena and Mesoscopic Devices in Microelectronics, Eds. I. O. Kulik and R. Ellialtioglu (Kluwer Acad. Publ., 2000), pp. 61-77.

10. A. Hahn, Phys. Rev. 31, 2816 (1985).

11. A. Hahn and K. Humpfner, Phys. Rev. B51, 3660 (1995).

12. U. Gunsenheimer and A. Hahn, Phys. B218, 141 (1996).

13. M. Belogolovski, M. Grajcar, P. Kus, A. Plecenik, S. Benacka, P. Seidel, Phys. Rev. B59, 9617 (1999).

14. V. A. Khlus and A. N. Omelyanchuk, Sov. Journ. Low Temp. Phys. 9, 189 (1983).

15. I. O. Kulik and A. N. Omelyanchuk, Zh. Exp. Theor. Fiz. 68, 2139 (1975) [Sov. Phys. JETP 41, 1071 (1975)]

16. I. O. Kulik, in Quantum Mesoscopic Phenomena and Mesoscopic Devices in Microelectronics, Eds. I. O. Kulik and R. Ellialtioglu (Kluwer Acad. Publ., 2000), pp. 3-22.

17. E. N. Bogachek, A. G. Sherbakov and U. Landman, Phys. Rev. B56, 14917 (1997). 
18. A. A. Abrikosov, Fundamentals of the Theory of Metals (in Russian), (Nauka Publ., Moscow, 1987); (North Holland, Amsterdam, 1988).

19. S. N. Artemenko, A. F. Volkov and A. V. Sergeev, J. Low Temp. Phys. 44, 405 (1981).

20. Yu. G. Bevza and A. V. Lukashenko, Fizika Nizkikh Temperatur 9, 368 (1983). 